IJMMS 32:12 (2002) 707-720

PII. S0161171202202355

http://ijmms.hindawi.com

(c) Hindawi Publishing Corp.

\title{
TRANSITIVITY IN UNIFORM APPROACH THEORY
}

\author{
Y. J. LEE and B. WINDELS
}

Received 26 February 2002

\begin{abstract}
We introduce a notion of transitivity for approach uniformities and approach uniform convergence spaces, yielding reflective subconstructs of AUnif and AUCS. Further, we investigate how these new categories are related to $\mathbf{u A C H Y}, \mathbf{u A C H Y} \mathbf{U}_{U}$, and $\mathbf{u M E T}$, and we show that these relationships are similar to those in the classical case.
\end{abstract}

2000 Mathematics Subject Classification: 18B30, 54E15.

1. Introduction. Since the first considerations on zero-dimensional spaces, by F. Hausdorff, and the original study of non-Archimedean metric spaces, by A. F. Monna, the amount of literature on transitive structures has become extensive. Transitivity turned out to be interesting in a wide range of fields (functional analysis, Boolean algebra, valuation rings, domain theory, and many others) which proves the great importance of the concept. Therefore, an investigation of this topic in the setting of uniform approach structures is inevitable.

This paper presents a transitivity condition for two important quantified uniform structures: one for approach uniformities (introduced in Lowen and Windels [5] as a quantification of Unif) and a related concept for approach uniform convergence spaces (introduced in Windels [7] as a quantification of UCS). These definitions in turn yield different transitivity concepts in the setting of approach Cauchy spaces (introduced in Lowen and Lee [4] as a quantification of CHY).

Although the categories UCS and CHY are well known to be Cartesian closed (see Lee [3] and Bentley et al. [1], respectively), the associated quantified structures yield categories which do not share this property; the triangle inequality-like axiom turned out to be the essential problem. One possible solution, which is discussed in [7], is to drop this particular axiom. Alternatively, we can demand a stronger (non-Archimedean) triangle inequality to be fulfilled: in the case of Cauchy spaces, this approach leads to the Cartesian closed category $\mathbf{u A C H Y}$ (see [4]). In this paper, we will pursue the same method for uniform convergence spaces.

For any set $X$, we denote the set of all filters on $X$ by $\mathscr{F}(X)$. The filter generated by a filter basis $\mathscr{B}$ is denoted by [ $\mathscr{B}]$. In particular, the point filter generated by the set $\{x\}$ is denoted by $\dot{x}$. If $\mathscr{F}, \mathscr{G} \in \mathscr{F}(X)$, then $\mathscr{F} \times \mathscr{G}=[\{F \times G: F \in \mathscr{F}, G \in \mathscr{G}\}]$. If $f: X \rightarrow Y$ is a map and $\mathscr{F} \in \mathscr{F}(X)$, then $f(\mathscr{F})=[\{f(F): F \in \mathscr{F}\}]$. If $\Phi \in \mathscr{F}(X \times X)$, then $\Phi^{-1}=\left\{U^{-1}: U \in \Phi\right\}$, where $U^{-1}=\{(y, x) \in X \times X:(x, y) \in U\}$. If $\Phi, \Psi \in \mathscr{F}(X \times X)$, then $\Phi \circ \Psi=[\{U \circ V: U \in \Phi, V \in \Psi\}]$, provided that every $U \circ V=\{(x, y) \in X \times X$ : there exists $z \in X$ such that $(x, z) \in U$ and $(z, y) \in V\}$ is not empty; whenever this notation is used, we will tacitly assume this condition to be fulfilled. 
Recall from [8] that a semi-uniform convergence structure $\mathbb{L}$ on a set $X$ is a collection of filters on $X \times X$ such that

(UCS1) $\dot{x} \times \dot{x} \in \mathbb{L}$ for all $x \in X$,

(UCS2) if $\Phi \in \mathbb{L}$ and $\Phi \subset \Psi$, then $\Psi \in \mathbb{L}$,

(UCS3) if $\Phi, \Psi \in \mathbb{L}$, then $\Phi \cap \Psi \in \mathbb{L}$,

(UCS4) if $\Phi \in \mathbb{L}$, then $\Phi^{-1} \in \mathbb{L}$.

The collection $\mathbb{L}$ is called a uniform convergence structure if it also satisfies the supplementary condition

(UCS5) if $\Phi, \Psi \in \mathbb{L}$, then $\Phi \circ \Psi \in \mathbb{L}$.

The pair $(X, \mathbb{L})$ is called a uniform convergence space.

For any semi-uniform convergence spaces $(X, \mathbb{L})$ and $(Y, \mathbb{K})$, a map $f: X \rightarrow Y$ is called uniformly continuous if $\Phi \in \mathbb{L}$ implies $(f \times f)(\Phi) \in \mathbb{K}$. Let UCS denote the category of uniform convergence spaces and uniformly continuous maps.

2. The category uAUCS. In this section, we introduce a notion of transitivity for approach uniform convergence structures. Recall from Windels [7] that an approach uniform convergence structure on a set $X$ is a map $\eta: \mathscr{F}(X \times X) \rightarrow[0, \infty]$ satisfying the following conditions: for all $x \in X$ and all $\Phi, \Psi \in \mathscr{F}(X \times X)$,

(AUCS1) $\eta(\dot{x} \times \dot{x})=0$

(AUCS2) $\Phi \subset \Psi$ implies $\eta(\Phi) \geq \eta(\Psi)$,

(AUCS3) $\eta(\Phi \cap \Psi)=\eta(\Phi) \vee \eta(\Psi)$,

(AUCS4) $\eta\left(\Phi^{-1}\right)=\eta(\Phi)$,

(AUCS5) $\eta(\Phi \circ \Psi) \leq \eta(\Phi)+\eta(\Psi)$.

Alternatively, such a structure can be described by a uniform convergence tower $\left(\mathbb{L}_{\varepsilon}\right)_{\varepsilon \in \mathbb{R}^{+}}\left(\right.$or $\left.\left(\mathbb{L}_{\varepsilon}\right)_{\varepsilon}\right)$, that is a collection of semi-uniform convergence structures $\mathbb{L}_{\varepsilon}$ on $X$ such that

(UCT1) if $\varepsilon, \varepsilon^{\prime} \in \mathbb{R}^{+}$and $\Phi \in \mathbb{R}_{\varepsilon}, \Psi \in \mathbb{L}_{\varepsilon^{\prime}}$, then $\Phi \circ \Psi \in \mathbb{L}_{\varepsilon+\varepsilon^{\prime}}$,

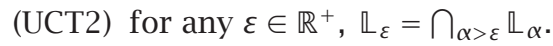

The equivalence is shown by considering $\mathbb{L}_{\varepsilon}=\{\mathscr{F} \in \mathscr{F}(X \times X): \eta(\mathscr{F}) \leq \varepsilon\}$ and $\eta(\mathscr{F})=$ $\min \left\{\varepsilon \in \mathbb{R}^{+}: \mathscr{F}_{\mathcal{F}} \in \mathbb{L}_{\varepsilon}\right\}$. The pair $(X, \eta)$ (or, equivalently, the pair $\left(X,\left(\mathbb{L}_{\varepsilon}\right)_{\varepsilon \in \mathbb{R}^{+}}\right)$) is called an approach uniform convergence space (AUC-space for short).

Given AUC-spaces $(X, \eta)$ and $\left(Y, \eta^{\prime}\right)$ with uniform convergence towers $\left(\mathbb{L}_{\varepsilon}\right)_{\varepsilon}$ and $\left(\mathbb{K}_{\varepsilon}\right)_{\varepsilon}$, respectively, a map $f: X \rightarrow Y$ is called a uniform contraction if one of the following equivalent conditions is satisfied:

(1) $\eta^{\prime}((f \times f)(\Phi)) \leq \eta(\Phi)$ for all $\Phi \in \mathscr{F}(X \times X)$,

(2) for each $\varepsilon \in \mathbb{R}^{+}, f:\left(X, \mathbb{L}_{\varepsilon}\right) \rightarrow\left(Y, \mathbb{K}_{\varepsilon}\right)$ is uniformly continuous. Let AUCS denote the category of AUC-spaces and uniform contractions. For details, the reader is referred to [7].

Definition 2.1. Let $X$ be a set. An AUC-structure $\eta: \mathscr{F}(X \times X) \rightarrow[0, \infty]$ is called an ultra approach uniform convergence structure if it satisfies instead of (AUCS5) the stronger condition: (uAUCS5) if $\Phi, \Psi \in \mathscr{F}(X \times X)$, then $\eta(\Phi \circ \Psi) \leq \eta(\Phi) \vee \eta(\Psi)$.

The pair $(X, \eta)$ is called an ultra approach uniform convergence space (uAUC-space for short).

uAUC-spaces can be described by uniform convergence towers too. 
Proposition 2.2. Let $(X, \eta)$ be an AUC-space, and let $\left(\mathbb{L}_{\varepsilon}\right)_{\varepsilon \in \mathbb{R}^{+}}$denote its uniform convergence tower. Then, the following are equivalent:

(1) $(X, \eta)$ is an ultra approach uniform convergence space,

(2) for every $\varepsilon \in \mathbb{R}^{+}, \mathbb{L}_{\varepsilon}$ is a uniform convergence structure.

Let uAUCS denote the full subcategory of AUCS consisting of all uAUC-spaces.

THEOREM 2.3. The category UAUCS is a bireflective subcategory of AUCS.

Proof. For a family $\left(\left(X_{j}, \eta_{j}\right)\right)_{j \in J}$ of uAUC-spaces and a source $\left(X \stackrel{f_{j}}{\longrightarrow}\left(X_{j}, \eta_{j}\right)\right)_{j \in J}$ in AUCS, the initial approach uniform convergence structure $\eta: \mathscr{F}(X \times X) \rightarrow[0, \infty]$ on $X$ defined by

$$
\Phi \longmapsto \eta(\Phi)=\sup _{j \in J} \eta_{j}\left(\left(f_{j} \times f_{j}\right)(\Phi)\right)
$$

satisfies (uAUCS5). For this, let $\Phi, \Psi \in \mathscr{F}(X \times X)$ be such that there exists $\Phi \circ \Psi$, then for each $j \in J,\left(f_{j} \times f_{j}\right)(\Phi) \circ\left(f_{j} \times f_{j}\right)(\Psi)$ exists and

$$
\eta_{j}\left(\left(f_{j} \times f_{j}\right)(\Phi) \circ\left(f_{j} \times f_{j}\right)(\Psi)\right) \leq \eta_{j}\left(\left(f_{j} \times f_{j}\right)(\Phi)\right) \vee \eta_{j}\left(\left(f_{j} \times f_{j}\right)(\Psi)\right) .
$$

So uAUCS is initially closed in AUCS and since uAUCS contains all indiscrete objects, this proves the claim.

THEOREM 2.4. The category uAUCS is a topological construct.

Proof. This is an immediate consequence of Theorem 2.3 and [2, Theorem A.10].

Initial sources can be described by means of towers as well.

Proposition 2.5. Let $(X, \eta)$ and $\left(\left(X_{j}, \eta_{j}\right)\right)_{j \in J}$ be uAUC-spaces, and let $\left(\mathbb{L}_{\varepsilon}\right)_{\varepsilon}$ and $\left(\mathbb{L}_{\varepsilon}^{j}\right)_{\varepsilon}$ denote the respective towers. Then, the following are equivalent:

(1) $\left((X, \eta) \stackrel{f_{i}}{\rightarrow}\left(X_{j}, \eta_{j}\right)\right)_{j \in J}$ is initial (in AUCS),

(2) $\forall \varepsilon \in \mathbb{R}^{+}:\left(\left(X, \mathbb{L}_{\varepsilon}\right) \stackrel{f_{j}}{\longrightarrow}\left(X_{j}, \mathbb{L}_{\varepsilon}^{j}\right)\right)_{j \in J}$ is initial (in UCS).

Proof. For every $\varepsilon \in \mathbb{R}^{+}$, let $\mathbb{K}_{\varepsilon}$ be the initial uniform convergence structure for the source $\left(X \stackrel{f_{j}}{\longrightarrow}\left(X_{j}, \square_{\varepsilon}^{j}\right)\right)_{j \in J}$. Then, for any $\Phi \in \mathscr{F}(X \times X), \eta(\Phi)=\sup _{j \in J} \eta_{j}\left(\left(f_{j} \times f_{j}\right)(\Phi)\right)$ and thus we have

$$
\begin{aligned}
\mathbb{L}_{\varepsilon} & =\{\Phi \in \mathscr{F}(X \times X): \eta(\Phi) \leq \varepsilon\} \\
& =\left\{\Phi \in \mathscr{F}(X \times X): \eta_{j}\left(\left(f_{j} \times f_{j}\right)(\Phi)\right) \leq \varepsilon \forall j \in J\right\} \\
& =\left\{\Phi \in \mathscr{F}(X \times X):\left(f_{j} \times f_{j}\right)(\Phi) \in \mathbb{L}_{\varepsilon}^{j} \forall j \in J\right\} \\
& =\mathbb{K}_{\varepsilon},
\end{aligned}
$$

which proves the claim.

For any uAUC-spaces $(X, \eta)$ and $\left(Y, \eta^{\prime}\right)$, let $C(X, Y)$ be the set of all uniform contractions from $X$ to $Y$. Then, for any $\Phi \in \mathscr{F}(X \times X)$ and $\Theta \in \mathscr{F}(C(X, Y) \times C(X, Y))$, the set $\{H(A): A \in \Phi, H \in \Theta\}$, where $H(A)=\{(h(a), k(b)):(a, b) \in A,(h, k) \in H\}$ for 
each $A \in \Phi$ and $H \in \Theta$, forms a filter basis on $Y \times Y$. Let $\Theta(\Phi)$ be the filter on $Y \times Y$ generated by this basis and define a map $\eta^{*}: \mathscr{F}(C(X, Y) \times C(X, Y)) \rightarrow[0, \infty]$ by

$$
\Theta \longmapsto \eta^{*}(\Theta)=\inf \{\alpha: \alpha \in L(\Theta)\},
$$

where

$$
L(\Theta)=\left\{\alpha: \eta^{\prime}(\Theta(\Phi)) \leq \eta(\Phi) \vee \alpha \forall \Phi \in \mathscr{F}(X \times X)\right\} .
$$

Proposition 2.6. The map $\eta^{*}$ yields the coarsest uAUC-structure on $C(X, Y)$ with respect to which the evaluation map ev: $X \times C(X, Y) \rightarrow Y$ defined by $(x, f) \mapsto f(x)$ is a uniform contraction.

Proof. Clearly, $\eta^{*}$ is well defined. (AUCS1) follows from the inequality

$$
\eta^{\prime}((\dot{f} \times \dot{f})(\Phi))=\eta^{\prime}((f \times f)(\Phi)) \leq \eta(\Phi)
$$

for all $f \in C(X, Y), \Phi \in \mathscr{F}(X \times X)$ and (AUCS2) is trivial since $\Theta \subset \Theta^{\prime}$ in $\mathscr{F}(C(X, Y) \times$ $C(X, Y))$ implies $\Theta(\Phi) \leq \Theta^{\prime}(\Phi)$ in $\mathscr{F}(Y \times Y)$ for all $\Phi \in \mathscr{F}(X \times X)$. For (AUCS3), let $\Theta, \Theta^{\prime} \in \mathscr{F}(C(X, Y) \times C(X, Y))$. Then

$$
\begin{aligned}
\eta^{*}\left(\Theta \cap \Theta^{\prime}\right) & =\inf \left\{\alpha: \eta^{\prime}\left(\left(\Theta \cap \Theta^{\prime}\right)(\Phi)\right) \leq \eta(\Phi) \vee \alpha \forall \mathscr{F} \in \mathscr{F}(X)\right\}, \\
\eta^{\prime}\left(\Theta \cap \Theta^{\prime}\right)(\Phi) & =\eta^{\prime}\left(\Theta(\Phi) \cap \Theta^{\prime}(\Phi)\right)=\eta^{\prime}(\Theta(\Phi)) \vee \eta^{\prime}\left(\Theta^{\prime}(\Phi)\right) .
\end{aligned}
$$

So $\eta^{*}\left(\Theta \cap \Theta^{\prime}\right) \leq \eta^{*}(\Theta) \vee \eta^{*}\left(\Theta^{\prime}\right)$ and the converse follows from (AUCS2). Since for any $\Theta \in \mathscr{F}(C(X, Y) \times C(X, Y))$ and $\Phi \in \mathscr{F}(X \times X)$ it holds that $\Theta^{-1}(\Phi)=\left(\Theta\left(\Phi^{-1}\right)\right)^{-1}$, (AUCS4) is immediate. Finally, let $\Theta, \Theta^{\prime} \in \mathscr{F}(C(X, Y) \times C(X, Y))$ be such that there exist $\Theta \circ \Theta^{\prime}$ and $\Phi \in \mathscr{F}(X \times X)$. Then for any $H \in \Theta, K \in \Theta^{\prime}$, and $A \in \Phi$, it holds that $(H \circ K)(A) \subseteq H(A) \circ K\left(A^{-1} \circ A\right)$ and hence

$$
\Theta(\Phi) \circ \Theta^{\prime}\left(\Phi^{-1} \circ \Phi\right) \subset\left(\Theta \circ \Theta^{\prime}\right)(\Phi) .
$$

So (uAUCS5) is fulfilled, consequently $\eta^{*}$ is an uAUC-structure on $C(X, Y)$. Since for any $\Psi \in \mathscr{F}((X \times C(X, Y)) \times(X \times C(X, Y)))$,

$$
\begin{aligned}
\eta^{\prime}((\mathrm{ev} \times \mathrm{ev})(\Psi)) & \leq \eta^{\prime}\left((\mathrm{ev} \times \mathrm{ev})\left(\left(\pi_{1} \times \pi_{1}\right)(\Psi) \times\left(\pi_{2} \times \pi_{2}\right)(\Psi)\right)\right) \\
& =\eta^{\prime}\left(\left(\pi_{2} \times \pi_{2}\right)(\Psi)\left(\left(\pi_{1} \times \pi_{1}\right)(\Psi)\right)\right) \\
& \leq \eta\left(\left(\pi_{1} \times \pi_{1}\right)(\Psi)\right) \vee \eta^{*}\left(\left(\pi_{2} \times \pi_{2}\right)(\Psi)\right) \\
& =\left(\eta \times \eta^{*}\right)(\Psi),
\end{aligned}
$$

where $\pi_{1}$ and $\pi_{2}$ are the canonical projection maps from $X \times C(X, Y)$ to $X$ and $C(X, Y)$, respectively, the map ev: $X \times C(X, Y) \rightarrow Y$ is a uniform contraction with respect to $\eta^{*}$. Let $\eta_{*}$ be another uAUC-structure on $C(X, Y)$ with respect to which ev is a uniform contraction. Then for all $\Phi \in \mathscr{F}(X \times X)$ and $\Theta \in \mathscr{F}(C(X, Y) \times C(X, Y))$, we have

$$
\eta^{\prime}((\mathrm{ev} \times \mathrm{ev})(\Phi \times \Theta))=\eta^{\prime}(\Theta(\Phi)) \leq \eta(\Phi) \vee \eta_{*}(\Theta),
$$

consequently, $\eta_{*}(\Theta) \in L(\Theta)$ for all $\Theta \in \mathscr{F}(C(X, Y) \times C(X, Y))$. So $\eta^{*}(\Theta) \leq \eta_{*}(\Theta)$ for all $\Theta \in \mathscr{F}(C(X, Y) \times C(X, Y))$ and hence we have the result. 
Proposition 2.7. Let $(X, \eta),\left(Y, \eta^{\prime}\right)$, and $\left(Z, \eta^{\prime \prime}\right)$ be uAUC-spaces and let $f: X \times Z \rightarrow$ $Y$ be a uniform contraction. Then there exists a unique uniform contraction $\hat{f}: Z \rightarrow$ $C(X, Y)$ such that $\mathrm{ev} \circ\left(1_{X} \times \hat{f}\right)=f$.

Proof. Define a map $\hat{f}: Z \rightarrow C(X, Y)$ by

$$
\begin{aligned}
& z \longmapsto \hat{f}(z): X \longrightarrow Y, \\
& x \longmapsto \hat{f}(z)(x)=f(x, z) .
\end{aligned}
$$

Then for each $z \in Z, \hat{f}(z)=f \circ\left(1_{X} \times[z]\right)$, where $[z]: X \rightarrow Z$ is a map defined by $x \mapsto z$ for all $x \in X$. Since the identity map, the constant map, and the composition of uniform contractions are uniform contractions, $\hat{f}$ is a uniform contraction and hence the map $\hat{f}$ is well defined. Furthermore, for any $\Phi \in \mathscr{F}(X \times X)$ and $\Psi \in \mathscr{F}(Z \times Z)$, we have

$$
\begin{aligned}
\eta^{\prime}(((\hat{f} \times \hat{f})(\Psi))(\Phi)) & =\eta^{\prime}((f \times f)(\Phi \times \Psi)) \\
& \leq\left(\eta \times \eta^{\prime \prime}\right)(\Phi \times \Psi) \\
& =\eta(\Phi) \vee \eta^{\prime \prime}(\Psi) .
\end{aligned}
$$

So $\eta^{*}((\hat{f} \times \hat{f})(\Psi)) \leq \eta^{\prime \prime}(\Psi)$ for all $\Psi \in \mathscr{F}(Z \times Z)$ and hence $\hat{f}$ is a uniform contraction. Clearly, ev $\circ\left(1_{X} \times \hat{f}\right)=f$ and such an $\hat{f}$ is unique.

Combining Propositions 2.6 and 2.7, we have the following theorem.

THEOREM 2.8. The category uAUCS is Cartesian closed.

For any uniform convergence space $(X, \mathbb{L})$, the map $\eta_{\mathbb{L}}: \mathscr{F}(X \times X) \rightarrow[0, \infty]$ defined by

$$
\Phi \longmapsto \eta_{\mathbb{L}}(\Phi)= \begin{cases}0 & \text { for } \Phi \in \mathbb{L}, \\ \infty & \text { for } \Phi \notin \mathbb{L}\end{cases}
$$

is clearly an uAUC-structure on $X$. Furthermore, for any uniform convergence spaces $(X, \mathbb{L})$ and $(Y, \mathbb{K})$, a map $f:(X, \mathbb{L}) \rightarrow(Y, \mathbb{K})$ is uniformly continuous if and only if $f:\left(X, \eta_{\mathbb{L}}\right) \rightarrow\left(Y, \eta_{\mathbb{K}}\right)$ is a uniform contraction.

So UCS is embedded as a full subcategory in uAUCS by the functor

$$
\begin{aligned}
\text { UCS } & \longrightarrow \text { uAUCS, } \\
(X, \mathbb{L}) & \longmapsto\left(X, \eta_{\mathbb{}}\right), \\
f & \longmapsto f,
\end{aligned}
$$

and analogously to [7, Proposition 11], we have the following proposition.

Proposition 2.9. An uAUC-space $(X, \eta)$ is a uniform convergence space if and only if $\eta(\mathscr{F}(X \times X)) \subseteq\{0, \infty\}$.

THEOREM 2.10. The category UCS is a bicoreflective subcategory of uAUCS. 
THEOREM 2.11. The category UCS is a bireflective subcategory of uAUCS.

3. The category AUnif ${ }_{U}$. In this section and in Section 4 we discuss two different notions of transitivity for approach uniformities. Recall from Lowen and Windels [5] that an approach uniformity on a set $X$, is an ideal $U$ of functions from $X \times X$ into $[0, \infty]$, satisfying the following conditions:

(AU1) for all $u \in U$, for all $x \in X: u(x, x)=0$,

(AU2) for all $v \in[0, \infty]^{X \times X}:\left(\forall \varepsilon>0, \forall N<\infty: \exists u_{\varepsilon}^{N} \in U\right.$ s.t. $\left.v \wedge N \leq u_{\varepsilon}^{N}+\varepsilon\right) \Rightarrow v \in$ ,

(AU3) for all $u \in U$, for all $N<\infty$, there exist $u^{N} \in U$ s.t. $\forall x, y, z \in X: u(x, z) \wedge N \leq$ $u^{N}(x, y)+u^{N}(y, z)$

(AU4) for all $u \in U: u^{s} \in \mathcal{U}$.

Equivalently, an approach uniformity can be described with a uniform tower, that is, a family of semi-uniformities $\left(U_{\varepsilon}\right)_{\varepsilon \in \mathbb{R}^{+}}\left(\right.$or $\left.\left(U_{\varepsilon}\right)_{\varepsilon}\right)$ on $X$, such that

(UT1) for all $\varepsilon, \varepsilon^{\prime} \in \mathbb{R}^{+}: u_{\varepsilon} \circ u_{\varepsilon^{\prime}} \supset \bigcup_{\varepsilon+\varepsilon^{\prime}}$,

(UT2) for all $\varepsilon \in \mathbb{R}^{+}: u_{\varepsilon}=\bigcup_{\alpha>\varepsilon} u_{\alpha}$.

The equivalence is shown by considering $U_{\varepsilon}=\{\{u<\alpha\}: \alpha>\varepsilon, u \in U\}$. The pair $(X, \cup)$ (or, equivalently, the pair $\left(X,\left(\cup_{\varepsilon}\right)_{\varepsilon \in \mathbb{R}^{+}}\right)$) is called an approach uniform space.

The function $f:(X, \mathcal{u}) \rightarrow\left(Y, u^{\prime}\right)$ is called a uniform contraction if and only if $u^{\prime} \in$ $u^{\prime}$ implies $u^{\prime} \circ(f \times f) \in U$. The category of approach uniform spaces and uniform contractions is denoted by AUnif. For details, the reader is referred to [5].

DEFINITION 3.1. An approach uniform space $\left(X,\left(U_{\varepsilon}\right)_{\varepsilon \in \mathbb{R}^{+}}\right)$satisfying the supplementary condition that every $u_{\varepsilon}$ is a uniformity, is called level-uniform.

This definition establishes a notion of transitivity in the sense of previous section. To be precise, if $\left(X,\left(U_{\varepsilon}\right)_{\varepsilon \in \mathbb{R}^{+}}\right)$is an approach uniform space, then the AUC-structure $\eta$ defined by $\eta(\Phi)=\min \left\{\varepsilon \in \mathbb{R}^{+}: \Phi \supset \mathcal{U}_{\varepsilon}\right\}$ is an uAUC-structure if and only if $\left(U_{\varepsilon}\right)_{\varepsilon}$ is level-uniform.

Let AUnif ${ }_{U}$ denote the full subcategory of AUnif consisting of all level-uniform approach uniform spaces (for short, AUnif $_{U}$-spaces). Level-uniform spaces can be characterized nicely by ideals of functions too.

Proposition 3.2. Let $(X, \mathcal{U})$ be an approach uniform space. Then the following are equivalent:

(1) $(X, \mathcal{U})$ is level-uniform,

(2) $U$ has a basis $\mathscr{B}$ such that for all $u \in \mathscr{B}$, for all $N<\infty$, there exist $u^{N} \in \mathscr{B}$, for all $x, y, z \in X: u(x, z) \wedge N \leq u^{N}(x, y) \vee u^{N}(y, z)$,

(3) for all $u \in U$, for all $\varepsilon>0$, for all $N<\infty$, there exist $u_{\varepsilon}^{N} \in \mathcal{U}$, for all $x, y, z \in X$ : $u(x, z) \wedge N \leq u_{\varepsilon}^{N}(x, y) \vee u_{\varepsilon}^{N}(y, z)+\varepsilon$.

Proof. In order to prove $(1) \Rightarrow(2)$, consider any $u=\inf _{i=1}^{n}\left(\alpha_{i-1}+\theta_{U_{i}}\right)$, which form a basis for $U$ (see [5]). Choose $V_{i} \in U_{\alpha_{i}}$ such that $V_{i} \circ V_{i} \subset U_{i}(i=1, \ldots, n)$ and $V_{1} \subset V_{2} \subset$ $\cdots \subset V_{n}$. Let $v=\inf _{i=1}^{n}\left(\alpha_{i-1}+\theta_{V_{i}}\right)$. Now suppose $v(x, y)=\alpha_{i-1}$ and $v(y, z)=\alpha_{j-1}$. Then $(x, y) \in V_{i} \subset V_{i \vee j}$ and $(y, z) \in V_{j} \subset V_{i \vee j}$, consequently, $(x, z) \in U_{i \vee j}$. Therefore $u(x, z) \leq \alpha_{i \vee j-1}=\alpha_{i-1} \vee \alpha_{j-1}=v(x, y) \vee v(y, z)$. The fact that (2) $\Rightarrow(3)$ is immediate. To prove that (3) $\Rightarrow(1)$, let $u \in \mathcal{U}$ bounded, $\varepsilon \in \mathbb{R}^{+}$, and $\alpha>\varepsilon$. By (3), there is some 
$v \in U$ such that for all $x, y, z \in X: u(x, z) \leq v(x, y) \vee v(y, z)+(\alpha-\varepsilon) / 2$. Then

$$
\left\{v<\frac{\alpha+\varepsilon}{2}\right\} \circ\left\{v<\frac{\alpha+\varepsilon}{2}\right\} \subset\{u<\alpha\}
$$

With every level-uniform approach uniform space $\left(X,\left(\mathcal{U}_{\varepsilon}\right)_{\varepsilon}\right)$, we can associate an uAUC-structure $\eta$ on $X$ defined by $\eta(\Phi)=\min \left\{\varepsilon \in \mathbb{R}^{+}: \Phi \supset \mathcal{U}_{\varepsilon}\right\}$. This procedure yields an embedding of AUnif $_{\mathrm{U}}$ into $\mathbf{u A U C S}$.

THEOREM 3.3. The category AUnif $\mathrm{U}_{\mathrm{U}}$ is a bireflective subcategory of uAUCS.

Proof. Let $\left((X, \eta) \stackrel{f_{j}}{\longrightarrow}\left(X_{j}, \eta_{j}\right)\right)_{j \in J}$ be an initial source in uAUCS, and suppose that every $\left(X_{j}, \eta_{j}\right)$ is level-uniform. If $\left(\mathbb{L}_{\varepsilon}\right)_{\varepsilon}$ is the tower of $\eta$ and for all $j \in J:\left(\mathbb{L}_{\varepsilon}^{j}\right)_{\varepsilon}$ is the tower of $\eta_{j}$, then, by Proposition 2.5, for all $\varepsilon \in \mathbb{R}^{+}:\left(\left(X, \mathbb{L}_{\varepsilon}\right) \stackrel{f_{j}}{\rightarrow}\left(X_{j}, \mathbb{L}_{\varepsilon}^{j}\right)\right)_{j \in J}$ is initial, and since Unif is a reflective subcategory of UCS, every $\left(X, \mathbb{L}_{\varepsilon}\right)$ is level-uniform. Conse-

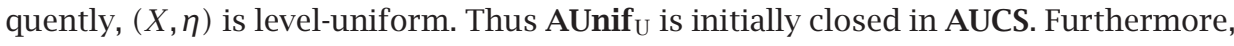
since $\mathrm{AUnif}_{\mathrm{U}}$ contains all indiscrete objects, we have the result.

Proposition 3.4. The category AUnif $_{\mathrm{U}}$ is a topological construct.

Proof. This is an immediate consequence of Theorem 3.3 and [2, Theorem A.10].

The category AUnif $\mathrm{U}_{\mathrm{U}}$ is not Cartesian closed, since it contains Unif both reflectively and coreflectively.

4. The category tAUnif. Since AUnif contains both the category of uniform spaces and the category of pseudo-metric spaces, it is natural to seek a subcategory of AUnif that generalizes the notions of transitive uniform spaces and ultra-metric spaces. Recall that a uniform space $(X, U)$ is called transitive if $u$ has a basis of entourages $U$ with the property that $U \circ U=U$. A pseudo-metric $d$ on $X$ is called an ultra-pseudometric (or non-Archimedean pseudo-metric) if $d$ satisfies the strong triangle inequality $d(x, z) \leq d(x, y) \vee d(y, z)$ for every $x, y, z \in X$.

Every approach uniformity induced by a transitive uniformity or by an ultra-metric is level-uniform, but not vice versa. In fact, every uniformly generated approach uniformity is level-uniform. This section establishes a stronger notion of transitivity for approach uniformities, in order to eliminate this disadvantage. Since every approach uniformity has a basis of pseudo-metrics, it seems natural to adopt the following definition.

DEFINITION 4.1. An approach uniform space $(X, \mathcal{U})$ is called transitive if $u$ has a basis consisting of ultra-pseudo-metrics.

Transitive approach uniformities can be described nicely in terms of uniform towers too.

Proposition 4.2. Let $(X, \mathcal{U})$ be an approach uniform space with a uniform tower $\left(U_{\varepsilon}\right)_{\varepsilon \in \mathbb{R}^{+}}$. Then the following are equivalent:

(1) $(X, \mathcal{U})$ is transitive,

(2) for every $\varepsilon \in \mathbb{R}^{+}, u_{\varepsilon}$ is a transitive uniformity. 
Proof. To see that $(1) \Rightarrow(2)$, notice that

$$
\{\{d<\alpha\}: \alpha>\varepsilon, d \in \mathcal{U}, d \text { ultra-pseudo-metric }\}
$$

is a transitive basis for $\mho_{\varepsilon}$. Conversely, if every $u_{\varepsilon}$ is a transitive uniformity, then (by [5, Lemma 2.7]) we know that

$$
\begin{array}{r}
\mathscr{B}=\left\{\inf _{i=1}^{n}\left(\alpha_{i-1}+\theta_{U_{i}}\right) \mid\left\{\alpha_{0}, \ldots, \alpha_{n}\right\} \delta \text {-net on }\left[0, \alpha_{n}\right],\right. \\
\left.\forall i \in\{1, \ldots, n\}: U_{i} \in \mathcal{U}_{\alpha_{i}} \text { and } U_{i} \circ U_{i}=U_{i}\right\}
\end{array}
$$

is a basis for $u$. Now suppose $u \in \mathscr{B}$ and $u(x, y)=\alpha_{i-1}$ and $u(y, z)=\alpha_{j-1}$. Then $(x, y) \in U_{i}$ and $(y, z) \in U_{j}$ and consequently $(x, z) \in U_{i \vee j}$. Therefore $u(x, z) \leq$ $\alpha_{i \vee j-1}=\alpha_{i-1} \vee \alpha_{j-1}=u(x, y) \vee u(y, z)$. Thus $\mathscr{B}$ is a basis consisting of ultra-pseudometrics.

Let tAUnif denote the full subcategory of AUnif consisting of all transitive approach uniformities.

THEOREM 4.3. The category tAUnif is a reflective subcategory of AUnif $\mathrm{U}_{\mathrm{U}}$. Consequently, tAUnif is a topological construct.

Proof. Since AUnif ${ }_{U}$ is a reflective subcategory of AUCS, initial structures in both categories are the same. Therefore the same argument as for Theorems 3.3 and Proposition 3.4 can be used.

Transitive approach uniformities generalize the notions of transitive uniformity and ultra-pseudo-metric.

Proposition 4.4. Let $(X, \mho)$ be a principal approach uniform space, that is, for all $\varepsilon \in \mathbb{R}^{+}, \cup_{\varepsilon}=U_{0}$. Then the following are equivalent:

(1) $(X, \mathcal{U})$ is transitive,

(2) $u_{0}$ is transitive.

Proof. By virtue of Proposition 4.2, this is evident.

Proposition 4.5. Let $(X, \mathcal{U})$ be a metric approach uniform space, that is, $\mathcal{U}=\{u$ : $u \leq d\}$ for some pseudo-metric $d$ on $X$. Then the following are equivalent:

(1) $(X, \mathcal{U})$ is transitive,

(2) $d$ is an ultra-pseudo-metric.

Proof. To see that $(1) \Rightarrow(2)$, suppose that $\mathscr{B}$ is a basis for $\mathscr{U}$ consisting of ultrapseudo-metrics. Then $d=\sup _{u \in \mathscr{乃}} u$, and therefore $d$ is an ultra-pseudo-metric too. The converse is trivial, since $\{d\}$ is a basis for $\mathcal{u}$.

The categories of ultra-pseudo-metric spaces and of transitive uniform spaces are nicely embedded in tAUnif, analogously to the classical case.

THEOREM 4.6. The category tUnif is a bireflective and bicoreflective subcategory of tAUnif. The category uMET is a bicoreflective subcategory of tAUnif. 
Therefore, we have the following diagram:

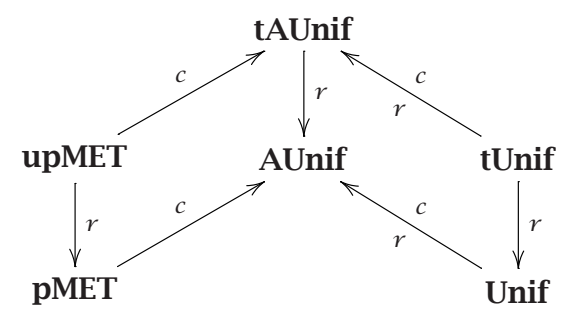

The category tAUnif is not Cartesian closed, since it contains tUnif both reflectively and coreflectively, and tUnif is not Cartesian closed (in fact, any reflective subcategory of Unif containing a nondiscrete object is not Cartesian closed).

5. Embedding UACHY in uAUCS. Recall from Lee and Lowen [4] that a function $\gamma: \mathscr{F}(X) \rightarrow[0, \infty]$ is called an ultra approach Cauchy structure (for short, uACHYstructure) on $X$ if it satisfies the following conditions:

(AF1) $\gamma(\dot{x})=0$ for all $x \in X$,

(AF2) if $\mathscr{F}, \mathscr{G} \in \mathscr{F}(X)$ and $\mathscr{F} \subset \mathscr{G}$, then $\gamma(\mathscr{F}) \geq \gamma(\mathscr{G})$, $(\mathrm{UACHY})$ if $\mathscr{F}, \mathscr{G} \in \mathscr{F}(X)$ and $\exists \mathscr{F} \vee \mathscr{G}$, then $\gamma(\mathscr{F} \cap \mathscr{G}) \leq \gamma(\mathscr{F}) \vee \gamma(\mathscr{G})$.

The pair $(X, \gamma)$ is called an ultra approach Cauchy space (for short, uACHY-space).

For any set $X$ and $\Phi \in \mathscr{F}(X \times X)$, let $\beta(\Phi)$ be the collection of all finite families $\left(\mathscr{F}_{j}\right)_{j=1}^{n} \subseteq \mathscr{F}_{(}(X)$ such that $\bigcap_{j=1}^{n}\left(\mathscr{F}_{j} \times \mathscr{F}_{j}\right) \subset \Phi$.

For any uACHY-space $(X, \gamma)$, define a map $\eta_{\gamma}: \mathscr{F}(X \times X) \rightarrow[0, \infty]$ by

$$
\Phi \longmapsto \eta_{\gamma}(\Phi)=\inf \left\{\sup _{j=1}^{n} \gamma\left(\mathscr{F}_{j}\right):\left(\mathscr{F}_{j}\right)_{j=1}^{n} \in \beta(\Phi)\right\} .
$$

Proposition 5.1. For any $u A U C H Y$-space $(X, \gamma)$, the map $\eta_{\gamma}$ is an $u A U C$-structure on $X$.

Proof. (AUCS1)-(AUCS4) are routine. To show that $\eta_{\gamma}$ fulfills (uAUCS5), let $\Phi, \Psi \in$ $\mathscr{F}(X \times X)$ be such that there exist $\Phi \circ \Psi$ and take any $\left(\mathscr{F}_{i}\right)_{i=1}^{n} \in \beta(\Phi),\left(\mathscr{G}_{j}\right)_{j=1}^{m} \in \beta(\Psi)$. Since $\Phi \circ \Psi$ exists, then there exists at least one pair of indices $\left(i_{0}, j_{0}\right)$ such that $\mathscr{F}_{i_{0}} \vee \mathscr{G}_{j_{0}}$ exists. Take all the pairs $\left(i_{k}, j_{k}\right)$ such that $\mathscr{F}_{i_{k}} \vee \mathscr{G}_{j_{k}}$ exists.

Then

$$
\begin{aligned}
\Phi \circ \Psi> & \left(\bigcap_{i=1}^{n}\left(\mathscr{F}_{i} \times \mathscr{F}_{i}\right)\right) \circ\left(\bigcap_{j=1}^{m}\left(\mathscr{G}_{j} \times \mathscr{G}_{j}\right)\right) \\
& =\bigcap_{\substack{i=1, \ldots, n \\
j=1, \ldots, m}}\left(\left(\mathscr{F}_{i} \times \mathscr{F}_{i}\right) \circ\left(\mathscr{G}_{j} \times \mathscr{G}_{j}\right)\right) \\
& =\bigcap_{k}\left(\left(\mathscr{F}_{i_{k}} \times \mathscr{F}_{i_{k}}\right) \circ\left(\mathscr{G}_{j_{k}} \times \mathscr{G}_{j_{k}}\right)\right) \\
& =\bigcap_{k}\left(\mathscr{F}_{i_{k}} \times \mathscr{G}_{j_{k}}\right) \\
& >\bigcap_{k}\left(\left(\mathscr{F}_{i_{k}} \cap \mathscr{G}_{j_{k}}\right) \times\left(\mathscr{F}_{i_{k}} \cap \mathscr{G}_{j_{k}}\right)\right) .
\end{aligned}
$$


So $\left(\mathscr{F}_{i_{k}} \cap \mathscr{G}_{j_{k}}\right)_{k} \in \beta(\Phi \circ \Psi)$ and since $\gamma\left(\mathscr{F}_{i_{k}} \cap \mathscr{G}_{j_{k}}\right)=\gamma\left(\mathscr{F}_{i_{k}}\right) \vee \gamma\left(\mathscr{G}_{j_{k}}\right)$ for each pair $\left(i_{k}, j_{k}\right)$, we have $\sup _{k} \gamma\left(\mathscr{F}_{i_{k}} \cap \mathscr{G}_{j_{k}}\right) \leq \sup _{i=1}^{n} \gamma\left(\mathscr{F}_{i}\right) \vee \sup _{j=1}^{m} \gamma\left(\mathscr{G}_{j}\right)$ and consequently $\eta_{\gamma}(\Phi \circ \Psi) \leq$ $\eta_{\gamma}(\Phi) \vee \eta_{\gamma}(\Psi)$.

Proposition 5.2. For any uACHY-spaces $(X, \gamma)$ and $\left(Y, \gamma^{\prime}\right)$, if a map $f:(X, \gamma) \rightarrow$ $\left(Y, \gamma^{\prime}\right)$ is a contraction, then $f:\left(X, \eta_{\gamma}\right) \rightarrow\left(Y, \eta_{\gamma^{\prime}}\right)$ is a uniform contraction.

Proof. For any $\Phi \in \mathscr{F}(X \times X)$ and $\left(\mathscr{F}_{j}\right)_{j=1}^{n} \in \beta(\Phi)$, we have $\left(f\left(\mathscr{F}_{j}\right)\right)_{j=1}^{n} \in \beta((f \times$ $f)(\Phi))$ and $\sup _{j=1}^{n} \gamma^{\prime}\left(f\left(\mathscr{F}_{j}\right)\right) \sup _{j=1}^{n} \gamma\left(\mathscr{F}_{j}\right)$.

Therefore, we have a functor

$$
\begin{aligned}
\text { uACHY } & \longrightarrow \text { uAUCS } \\
(X, \gamma) & \longmapsto\left(X, \eta_{\gamma}\right), \\
f & \longmapsto f .
\end{aligned}
$$

For any uAUC-space $(X, \eta)$, let $\gamma_{\eta}: \mathscr{F}(X) \rightarrow[0, \infty]$ be the map defined by

$$
\mathscr{F} \longmapsto \gamma_{\eta}(\mathscr{F})=\eta(\mathscr{F} \times \mathscr{F}) .
$$

Proposition 5.3. For any uAUC-space $(X, \eta)$, the pair $\left(X, \gamma_{\eta}\right)$ is an ultra approach Cauchy space.

Proof. (AF1) and (AF2) are immediate. For (uACHY) note that for any $\mathscr{F}, \mathscr{G} \in \mathscr{F}(X)$, it holds that $(\mathscr{F} \cap \mathscr{G}) \times(\mathscr{F} \cap \mathscr{G})=(\mathscr{F} \times \mathscr{F}) \cap(\mathscr{F} \times \mathscr{G}) \cap(\mathscr{G} \times \mathscr{F}) \cap(\mathscr{G} \times \mathscr{G})$ and if $\mathscr{F} \vee \mathscr{G}$ exists, then $(\mathscr{F} \times \mathscr{F}) \circ(\mathscr{G} \times \mathscr{G})=\mathscr{F} \times \mathscr{G}$. So for any $\mathscr{F}, \mathscr{G} \in \mathscr{F}(X)$ such that $\mathscr{F} \vee \mathscr{G}$ exists, we have $\gamma_{\eta}(\mathscr{F} \cap \mathscr{G})=\gamma_{\eta}(\mathscr{F}) \vee \gamma_{\eta}(\mathscr{G})$ by (AUCS3), (AUCS4), and (uAUCS5).

Proposition 5.4. For any uAUC-spaces $(X, \eta)$ and $\left(Y, \eta^{\prime}\right)$, if $f:(X, \eta) \rightarrow\left(Y, \eta^{\prime}\right)$ is a uniform contraction, then $f:\left(X, \gamma_{\eta}\right) \rightarrow\left(Y, \gamma_{\eta^{\prime}}\right)$ is a contraction.

So there exists a functor

$$
\begin{aligned}
\text { uAUCS } & \longrightarrow \text { uACHY, } \\
(X, \eta) & \longmapsto\left(X, \gamma_{\eta}\right), \\
f & \longmapsto f .
\end{aligned}
$$

Proposition 5.5. (1) For any uACHY-structure $\gamma$ on a set $X, \gamma=\gamma_{\eta_{\gamma}}$.

(2) For any uAUC-structure $\eta$ on $X, \eta \leq \eta_{\gamma_{\eta}}$.

PROoF. (1) For any $\mathscr{F}_{\mathscr{F}} \in \mathscr{F}_{(}(X)$, take any $\left(\mathscr{F}_{j}\right)_{j=1}^{n} \in \beta(\mathscr{F} \times \mathscr{F})$. Without loss of generality, we may assume $\mathscr{F}_{i} \vee \mathscr{F}_{j}$ does not exist for $i \neq j$ and hence we can take $\left(A_{j}\right)_{j=1}^{n}$ such that $A_{j} \in \mathscr{F}_{j}$ for each $j=1, \ldots, n$ and $A_{i} \cap A_{j}=\varnothing$ for $i \neq j$. Then there exists $F \in \mathscr{F}_{F}$ such that $F \times F \subseteq \bigcup_{j=1}^{n}\left(A_{j} \times A_{j}\right)$ and so $F \subseteq A_{k}$ for some $k \in\{1, \ldots, n\}$. Since $A_{j} \cap A_{k}=\varnothing$ for $j \neq k$, we get $\mathscr{F}_{k} \subset \mathscr{F}$ and consequently $\gamma\left(\mathscr{F}_{k}\right) \geq \gamma(\mathscr{F})$. Thus $\gamma \leq \gamma_{\eta_{\gamma}}$ and the converse is obvious.

(2) For any $\Phi \in \mathscr{F}(X \times X)$ and $\left(\mathscr{F}_{j}\right)_{j=1}^{n} \in \beta(\Phi)$, we have

$$
\sup _{j=1}^{n} \gamma_{\eta}\left(\mathscr{F}_{j}\right)=\sup _{j=1}^{n} \eta\left(\mathscr{F}_{j} \times \mathscr{F}_{j}\right)=\eta\left(\bigcap_{j=1}^{n}\left(\mathscr{F}_{j} \times \mathscr{F}_{j}\right)\right) \geq \eta(\Phi)
$$

and hence we have the result. 
THEOREM 5.6. The category $\mathrm{uACHY}$ is a bicoreflective subcategory of uAUCS.

Proof. By [6, Theorem 2.2.10], for any uAUC-space $(X, \eta)$

$$
1_{X}:\left(X, \eta_{\gamma_{\eta}}\right) \longrightarrow(X, \eta)
$$

is the $\mathbf{u A C H Y}$-bicoreflection.

6. The categories $\mathbf{u A C H Y} Y_{\mathrm{U}}$ and $\mathrm{uACHY}_{\mathrm{tU}}$. Throughout this section $(X, \mathcal{U})$ will be a level-uniform approach uniform space, and $\left(U_{\varepsilon}\right)_{\varepsilon}$ will denote its uniform tower. Then the map $\gamma_{\varkappa}: \mathscr{F}_{\mathcal{F}}(X) \rightarrow[0, \infty]$ defined by

$$
\mathscr{F} \longmapsto \gamma_{\text {ฯ }}(\mathscr{F})=\inf \left\{\varepsilon \in \mathbb{R}^{+}: \mathscr{F} \text { is a } u_{\varepsilon} \text {-Cauchy filter }\right\}
$$

is an ultra approach Cauchy structure on $X$. Conditions (AF1) and (AF2) are obvious and (uACHY) is immediate from (UT4) and the fact that each $\mho_{\varepsilon}$ is a uniform structure on $X$. We say that $\gamma_{\varkappa}$ and $U$ are compatible and $\gamma_{\varkappa}$ is called the uACHY-structure induced by $U$. Given a set $X$, an uACHY-structure $\gamma$ on $X$ is said to be approach uniformizable if there exists a compatible AUnif $_{\mathrm{U}}$-structure $U$ on $X$, that is, $\gamma=\gamma_{\varkappa}$ for some AUnif ${ }_{U}$-structure $U$ on $X$.

Let $\mathbf{U A C H Y}$ be the full subcategory of $\mathbf{u A C H Y}$ consisting of all approach uniformiz-

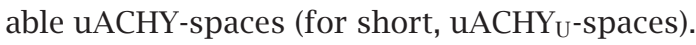

For any AUnif $\mathrm{U}_{U}$-space $(X, \mathcal{U})$, the pair $\left(X, \gamma_{\varkappa}\right)$ is a $\mathrm{uACHY}_{\mathrm{U}}$-space and if a map $f:(X, \mathcal{U}) \rightarrow\left(Y, \mathcal{U}^{\prime}\right)$ is a uniform contraction between AUnif $_{\mathrm{U}^{-}}$-spaces, then for any $\varepsilon \in \mathbb{R}^{+}$and $\mathscr{F} \in \mathscr{F}(X)$ such that $\mathscr{F}$ is a $u_{\varepsilon}$-Cauchy filter, $f(\mathscr{F})$ is a $u_{\varepsilon}^{\prime}$-Cauchy filter and so $\gamma_{\varkappa^{\prime}}(f(\mathscr{F})) \leq \gamma_{\text {น }}(\mathscr{F})$. Thus the map $f:\left(X, \gamma_{\varkappa}\right) \rightarrow\left(Y, \gamma_{\varkappa^{\prime}}\right)$ is a contraction between $\mathrm{uACHY}_{\mathrm{U}}$-spaces. Thus it defines a functor

$$
\begin{aligned}
\operatorname{AUnif}_{\mathrm{U}} & \longrightarrow \mathbf{u A C H Y}_{\mathrm{U}}, \\
(X, u) & \longmapsto\left(X, \gamma_{\text {थ }}\right), \\
f & \longmapsto f .
\end{aligned}
$$

Proposition 6.1. If $\mathcal{U}$ is the initial approach uniformity for a source

$$
\left(X \stackrel{f_{j}}{\longrightarrow}\left(X_{j}, \cup_{j}\right)\right)_{j \in J}
$$

in AUnif $_{\mathrm{U}}$ and $\gamma$ is the initial uACHY-structure for the induced source

$$
\left(X \stackrel{f_{j}}{\longrightarrow}\left(X_{j}, \gamma_{\cup_{j}}\right)\right)_{j \in J}
$$

in $\mathbf{u A C H Y}$, then $\gamma=\gamma_{\text {थ }}$.

Proof. Denote for every $j \in J$ the uniform tower of $u_{j}$ by $\left(u_{\varepsilon}^{j}\right)_{\varepsilon}$. For any $j \in J$, the map $f_{j}:\left(X, \cup_{u}\right) \rightarrow\left(X_{j}, \cup_{j}\right)$ is a uniform contraction and hence the induced map $f_{j}:\left(X, \gamma_{\vartheta_{u}}\right) \rightarrow\left(X_{j}, \gamma_{\iota_{j}}\right)$ is a contraction. So the map $1_{X}:\left(X, \gamma_{\text {ช }}\right) \rightarrow(X, \gamma)$ is a contraction by the initiality of $\gamma$ and we have $\gamma \leq \gamma_{\varkappa}$. For the converse, note that $\gamma: \mathscr{F}(X) \rightarrow$ $[0, \infty]$ is a map defined by

$$
\mathscr{F} \longmapsto \gamma(\mathscr{F})=\sup _{j \in J} \gamma_{\mho_{j}}\left(f_{j}(\mathscr{F})\right) .
$$


Let $\varepsilon \in \mathbb{R}^{+}$and $\mathscr{F} \in \mathscr{F}(X)$ be such that $\gamma(\mathscr{F}) \leq \varepsilon$. Then for each $j \in J, \gamma_{\cup_{j}}\left(f_{j}(\mathscr{F})\right) \leq \varepsilon$ and $f_{j}(\mathscr{F})$ is a $u_{\varepsilon}^{j}$-Cauchy filter on $X_{j}$. Thus $\mathscr{F}$ is a $u_{\varepsilon}$-Cauchy filter on $X$ and so $\gamma_{\text {थ }}(\mathscr{F}) \leq \varepsilon$. Therefore $\gamma \geq \gamma_{\text {थ }}$, which proves the claim.

For any uACHY $\mathrm{U}_{\mathrm{U}}$-space $(X, \gamma)$, let $U(\gamma)$ be the class of all AUnif $\mathrm{U}_{\mathrm{U}}$-uniform towers inducing $\gamma$.

THEOREM 6.2. The category $\mathrm{uACHY}_{\mathrm{U}}$ is a bireflective subcategory of uACHY.

Proof. For any family $\left(\left(X_{j}, \gamma_{j}\right)\right)_{j \in J}$ of $\mathrm{uACHY}_{\mathrm{U}}$-spaces, say $\gamma_{j}=\gamma_{{ }_{u_{j}}}$, and any source

$$
\left(X \stackrel{f_{j}}{\longrightarrow}\left(X_{j}, \gamma_{j}\right)\right)_{j \in J}
$$

in $\mathbf{u A C H Y}$, let $\gamma$ be the initial uACHY-structure on $X$ and let $u$ be the initial $\mathrm{AUnif}_{\mathrm{U}^{-}}$ uniform tower on $X$ for the source

$$
\left(X \stackrel{f_{j}}{\longrightarrow}\left(X_{j}, u_{j}\right)\right)_{j \in J} .
$$

Then $U$ induces $\gamma$ by Proposition 6.1 and hence $\mathbf{u A C H Y} \mathbf{Y}_{U}$ is initially closed in uACHY. Furthermore, since $\mathbf{u A C H Y} \mathbf{U}_{\mathrm{U}}$ contains all indiscrete objects, we have the result.

For any $\mathrm{uACHY} \mathrm{U}_{\mathrm{U}}$-space $(X, \gamma)$, let $\mho_{\gamma}$ be the initial AUnif $\mathrm{U}_{\mathrm{U}}$-structure with respect to the source $\left(X \stackrel{1_{X}}{\longrightarrow}\left(X, \cup_{)}\right)\right)_{u \in \vartheta(\gamma)}$. then $\vartheta_{\gamma}$ is the finest AUnif ${ }_{\mathrm{U}}$-structure on $X$ inducing $\gamma$ by Proposition 6.1.

Proposition 6.3. For any $u A C H Y_{U^{-}}$spaces $(X, \gamma)$ and $\left(Y, \gamma^{\prime}\right)$, if $f:(X, \gamma) \rightarrow\left(Y, \gamma^{\prime}\right)$ is a contraction, then $f:\left(X, \cup_{\gamma}\right) \rightarrow\left(Y, \cup_{\gamma^{\prime}}\right)$ is a uniform contraction between AUnif $U_{U^{-}}$ spaces.

Proof. For any uACHY $\mathrm{U}_{\mathrm{U}}$-space $(X, \gamma)$, let $C(X, \gamma)$ be the collection of all contractions from $(X, \gamma)$ to $\mathrm{uACHY}_{\mathrm{U}}$-spaces and let $\boldsymbol{U}$ be the initial AUnif $\mathrm{U}_{\mathrm{U}}$-structure on $X$ for the source

$$
\left(X \stackrel{g}{\longrightarrow}\left(Z, u_{\gamma^{\prime \prime}}\right)\right)_{g \in C(X, \gamma)}
$$

in AUnif $_{\mathrm{U}}$. Then $\gamma_{\varkappa}$ is the initial $\mathrm{uACHY}_{\mathrm{U}}$-structure on $X$ for the source

$$
\left(X \stackrel{g}{\longrightarrow}\left(Z, \gamma^{\prime \prime}\right)\right)_{g \in C(X, \gamma)}
$$

in $\mathbf{U A C H Y} \mathbf{Y}_{\mathrm{U}}$ and hence $1_{X}:(X, \gamma) \rightarrow\left(X, \gamma_{u}\right)$ is a contraction. For the converse, note that if a map $f:(X, \gamma) \rightarrow\left(Y, \gamma^{\prime}\right)$ is a contraction, then $f:\left(X, \gamma_{\varkappa}\right) \rightarrow\left(Y, \gamma^{\prime}\right)$ is a contraction. So by the fact that the identity map is a contraction, we get $1_{X}:\left(X, \gamma_{\text {थ }}\right) \rightarrow(X, \gamma)$ is a contraction. Therefore $u \in U(\gamma)$ and so $1_{X}:\left(X, \cup_{\gamma}\right) \rightarrow(X, u)$ is a uniform contraction. Thus, if $f:(X, \gamma) \rightarrow\left(Y, \gamma^{\prime}\right)$ is a contraction, then $f:(X, \mathcal{u}) \rightarrow\left(Y, \mathcal{U}_{\gamma^{\prime}}\right)$ is a uniform contraction and hence $f:\left(X, \cup_{\gamma}\right) \rightarrow\left(Y, \cup_{\gamma^{\prime}}\right)$ is a uniform contraction.

Hence there is a functor

$$
\begin{aligned}
\mathbf{u A C H Y}_{\mathrm{U}} & \longrightarrow \text { AUnif }_{\mathrm{U}}, \\
(X, \gamma) & \longmapsto\left(X, u_{\gamma}\right), \\
f & \longmapsto f .
\end{aligned}
$$


THEOREM 6.4. The category $\mathbf{u A C H Y}_{\mathrm{U}}$ is a bicoreflective subcategory of AUnif $_{\mathrm{U}}$.

Proof. For any uACHY $\mathrm{U}_{\mathrm{U}}$-structure $\gamma$ on a set $X$, we have $\gamma_{u_{\gamma}}=\gamma$ and for any AUnif $_{\mathrm{U}}$-structure $\boldsymbol{U}$ on $X, \boldsymbol{U}_{\text {ชथ }}$ is finer than $\boldsymbol{U}$. So by [6, Theorem 2.2.10], for any $\operatorname{AUnif}_{\mathrm{U}}$-space $(X, \mathcal{U})$

$$
1_{X}:\left(X, \text { น }_{\text {ชัน }}\right) \longrightarrow(X, \text { น })
$$

is the $\mathbf{u A C H Y} Y_{U}$-bicoreflection.

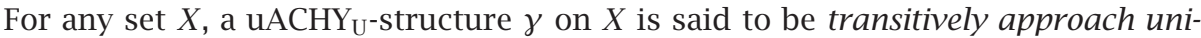
formizable if $\gamma$ is compatible with some transitive approach uniformity $U$ on $X$.

Let $\mathbf{u A C H Y}_{\mathrm{tU}}$ be the full subcategory of $\mathbf{u A C H Y}$ consisting of all transitive approach uniformizable uACHY-spaces (for short, $\mathrm{uACHY}_{\mathrm{tU}}$-spaces).

Since tAUnif is initially closed in AUnif $_{\mathrm{U}}$, we have the following theorem.

THEOREM 6.5. The category $\mathbf{u A C H Y} \mathrm{HU}_{\mathrm{tU}}$ is a bireflective subcategory of $\mathbf{u A C H Y}$.

For any $\mathrm{uACHY} \mathrm{tU}^{-}$-space $(X, \gamma)$, let $u_{\gamma}^{t}$ be the initial transitive approach uniformity

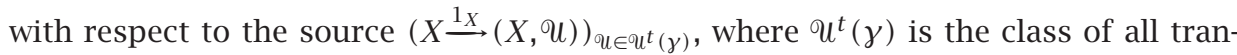
sitive approach uniformities inducing $\gamma$. Then $u_{\gamma}^{t}$ is the finest transitive approach uniformity on $X$ inducing $\gamma$. Clearly the restriction of the above two functors are well defined and we get the analogous result.

THEOREM 6.6. The category $\mathbf{u A C H Y}_{\mathrm{tU}}$ is a bicoreflective subcategory of tAUnif.

7. Categorical overview. Summarizing the results in foregoing sections, we obtain the following diagram:

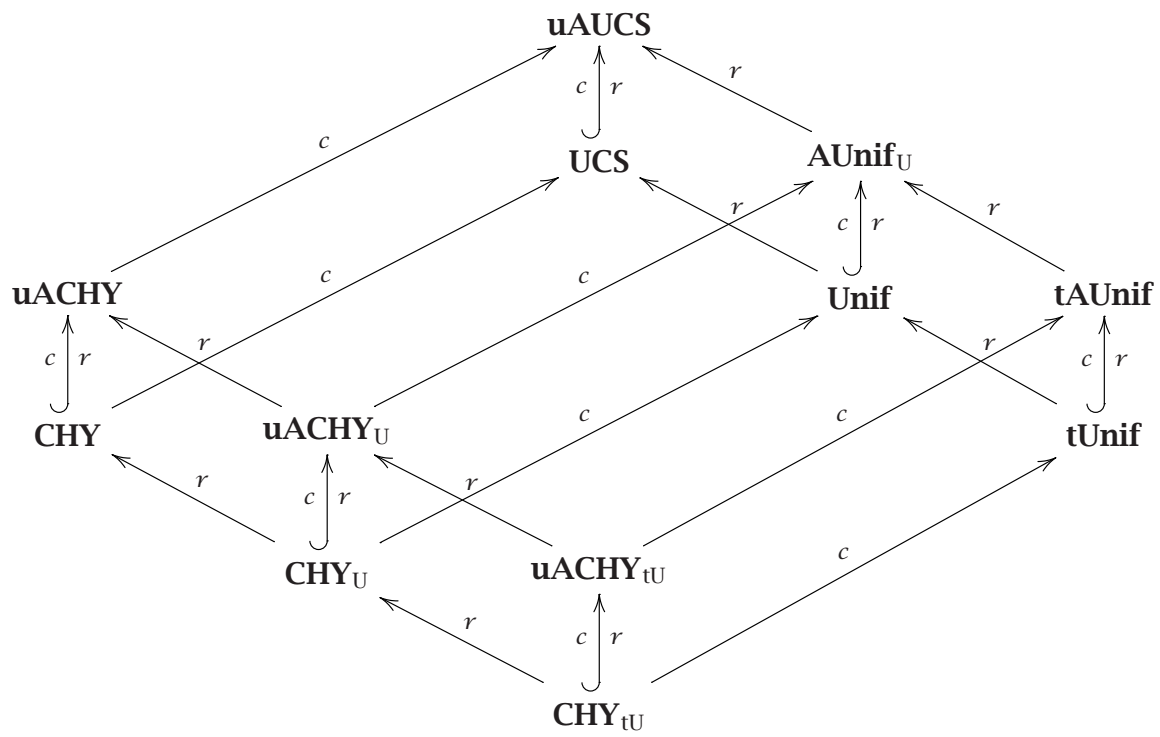


The categories tUnif and $\mathbf{C H Y}_{\mathrm{tU}}$ are the full subcategories of Unif and $\mathbf{C H Y}_{\mathrm{U}}$ whose objects are transitive uniform spaces and transitive uniformizable Cauchy spaces, respectively. These categories form a similar diagram as Unif and $\mathbf{C H Y}_{\mathrm{U}}$. At the end of Section 2, we showed that UCS is both reflectively and coreflectively embedded in uAUCS. The argument is representative for all upward arrows in the diagram.

\section{REFERENCES}

[1] H. L. Bentley, H. Herrlich, and E. Lowen-Colebunders, The category of Cauchy spaces is Cartesian closed, Topology Appl. 27 (1987), no. 2, 105-112.

[2] H. Herrlich, Topological structures, Topological Structures (Proc. Sympos. in Honour of Johannes de Groot (1914-1972), Amsterdam, 1973), Mathematical Centre Tracts, no. 52, Mathematisch Centrum, Amsterdam, 1974, pp. 59-122.

[3] R. S. Lee, The category of uniform convergence spaces is Cartesian closed, Bull. Austral. Math. Soc. 15 (1976), no. 3, 461-465.

[4] R. Lowen and Y. J. Lee, Approach theory in merotopic, Cauchy and convergence spaces. II, Acta Math. Hungar. 83 (1999), no. 3, 209-229.

[5] R. Lowen and B. Windels, AUnif: a common supercategory of pMET and Unif, Int. J. Math. Math. Sci. 21 (1998), no. 1, 1-18.

[6] G. Preuss, Theory of Topological Structures, Mathematics and Its Applications, vol. 39, D. Reidel Publishing, Dordrecht, 1988.

[7] B. Windels, Uniform approach theory, Ph.D. thesis, University of Antwerp, Belgium, 1997.

[8] O. Wyler, Filter space monads, regularity, completions, TOPO 72-General Topology and Its Applications (Proc. Second Pittsburgh Internat. Conf., Pittsburgh, Pa, 1972; dedicated to the memory of Johannes H. de Groot), Lecture Notes in Mathematics, vol. 378, Springer, Berlin, 1974, pp. 591-637.

\section{Y. J. LeE: Department of MATHematics, YonSei University, Seoul 120-749, KoreA}

B. Windels: Department of MATHEMATICS AND COMPUTER SCIENCE, RUCA, UNIVERSITY OF ANTWERP, GROENENBORGERLAAN 171, 2020 ANTWERP, BELGIUM 


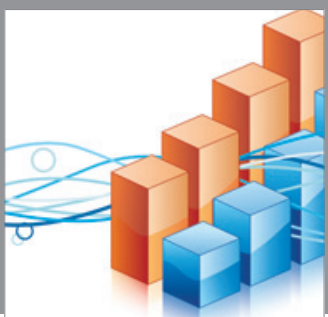

Advances in

Operations Research

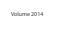

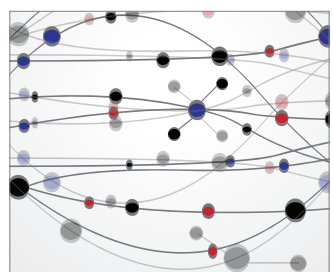

\section{The Scientific} World Journal
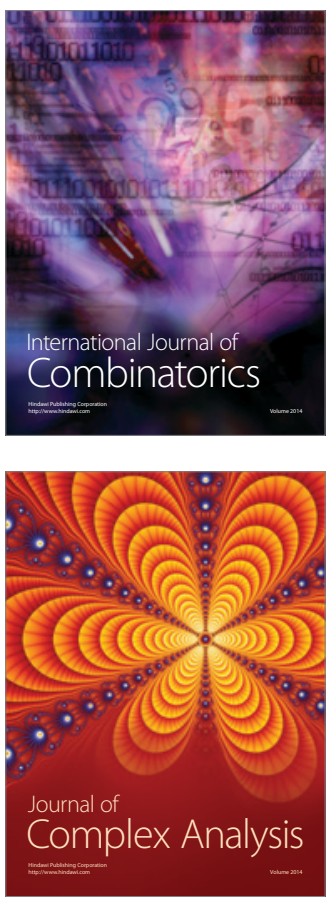

International Journal of

Mathematics and

Mathematical

Sciences
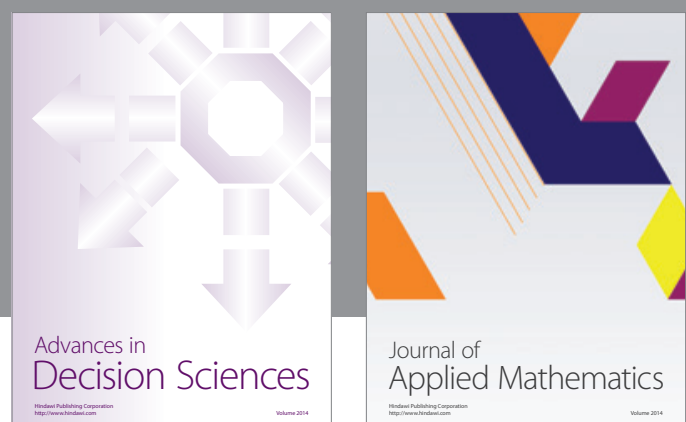

Journal of

Applied Mathematics
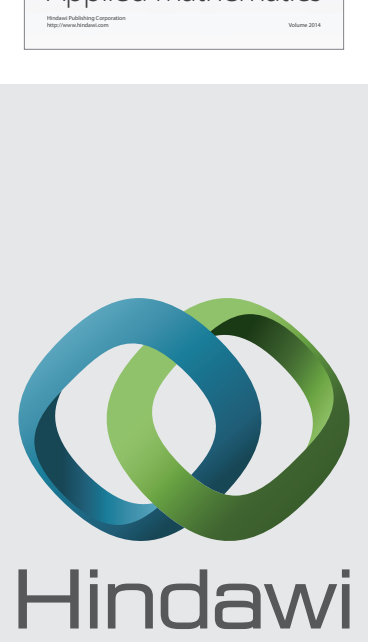

Submit your manuscripts at http://www.hindawi.com
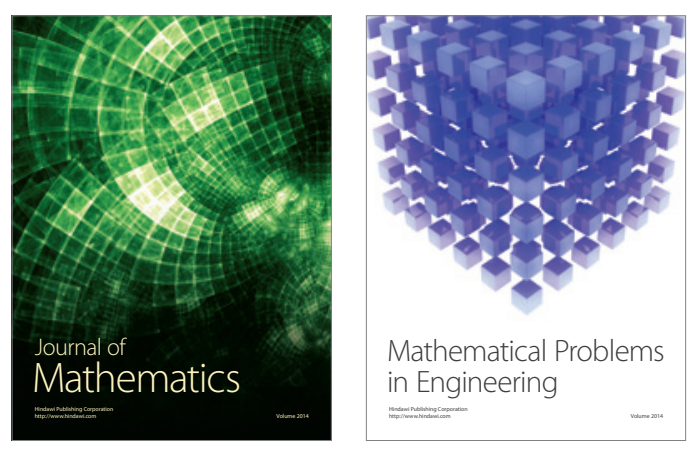

Mathematical Problems in Engineering
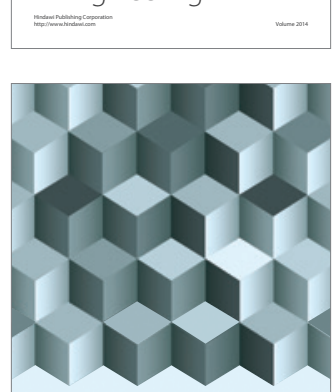

Journal of

Function Spaces
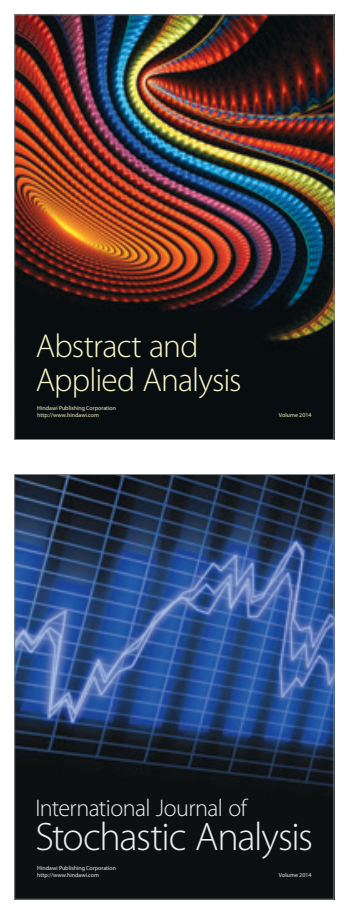

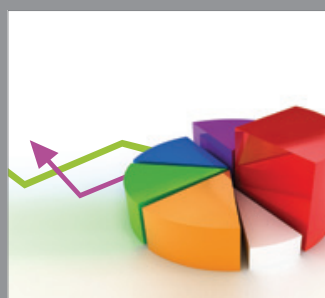

ournal of

Probability and Statistics

Promensencen
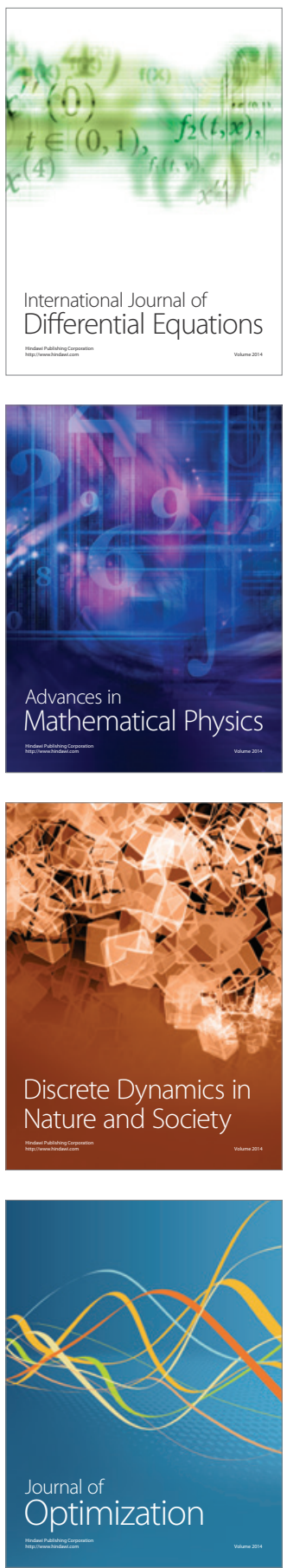